Check for updates

Cite this: RSC Adv., 2018, 8, 9120

\title{
Optical modulation of microfibers and application to ultrafast fiber lasers
}

\author{
Ruwei Zhao, (D) Guoru Li, Baitao Zhang and Jingliang He* \\ Microfibers with different waist diameters were prepared successfully by a flame-brushing technique. Their \\ saturable absorption properties were investigated. The non-saturable loss and modulation depth both \\ decreased with the increase of the diameter. According to the mode distribution of microfibers with \\ a waist diameter of $25 \mu \mathrm{m}$, it could be supposed that the evanescent field effect may be useful for \\ microfibers being saturable absorbers (SAs). Based on the $25 \mu \mathrm{m}$-diameter microfiber, an all-fiber- \\ structure mode-locked fiber laser was achieved successfully with long term stability in the span of \\ a week. The results indicated that microfibers with suitable diameters were excellent SAs. To the best of \\ our knowledge, this is first report on the usage of microfibers as a SA for building ultrafast fiber lasers.
}

Received 24th January 2018

Accepted 26th February 2018

DOI: $10.1039 / \mathrm{c} 8 \mathrm{ra00740c}$

rsc.li/rsc-advances
Optical modulation is one of crucial operations in photonics. ${ }^{14}$ Recently, two-dimensional materials (such as graphene, transition metal dichalcogenides, and black phosphorous) have been extensively studied in optical technology. By incorporating the materials onto microfiber via the strong evanescent filed, mode-locked fiber laser can be easily achieved, which provide the application in optical modulator of microfiber. ${ }^{15-17}$ However, whether the microfiber itself is competent as SA to realize ultrafast laser has not been reported yet.

In this work, the nonlinear optical properties of microfibers with different diameters have been experimentally studied. The microfibers were fabricated using flame-brushing technique. With the I-scan measurement, the saturable absorption property of microfiber was characterized. The experimental results displayed that the non-saturable loss and modulation depth both decrease with the increase of diameter of microfibers. What's more, based on a $25 \mu \mathrm{m}$-diameter microfiber, we have successfully realized an all-fiber-structure dual-wavelength mode-locked laser with the maximum average output power of $19 \mathrm{~mW}$. The spectrum with the peaks at 1593.9 and $1595.3 \mathrm{~nm}$ had full width half maximum (FWHM) of 0.64 and $0.72 \mathrm{~nm}$, respectively. The pulse repetition rate third-order harmonic mode-locked fiber laser was also achieved, just by increasing the pump power. Besides, the pulsed laser long-time stability in a span of a week was studied. The results proved microfiber with suitable diameter was an excellent saturable absorber. To the best of our knowledge, it is the first time to investigate the optical modulation of microfibers and application in the ultrafast fiber laser.

\section{Preparation and characterization of microfibers}

Compared to other manufacture of microfiber such as chemical growth and nano-imprint, flame-brushing technique yield 
microfibers with low surface roughness, large length and excellent diameter uniformity. ${ }^{\mathbf{1 1 8}}$ Here we fabricated the microfibers using flame-brushing technique as shown in the inset of Fig. 1(a). The bare standard SMFs were stretched in virtue of a flame. By adjusting the pulling distance, microfibers with the taper waist diameter of $25,38,55,65$, and $78 \mu \mathrm{m}$ were achieved. The transmission spectra of $25 \mu \mathrm{m}$-waist-diameter microfiber is shown in Fig. 1. Due to the scope limit of the pump source, the transmission spectra was measured in the range of 1530-1570 $\mathrm{nm}$. The result shows that the microfiber has a weak filtering effect, which may be useful for the generation of dual-wavelength laser. The scanning electron microscopy (SEM) was usually used to study the morphologies of sample. Here, Fig. 1(b) and (c) showed SEM image of microfiber with the waist diameter of $25 \mu \mathrm{m}$ using different scale bars, which proved high evenness degree and smooth surface of prepared microfibers. With a visible $455 \mathrm{~nm}$ laser guided through the $25 \mu \mathrm{m}$-diameter microfiber, it was clearly seen the evanescent field from scattered light as displayed in inset of Fig. 1. The microfiber was fixed on a slide glass by the adiabatic tape. The refractive index of microfiber and the slide glass is 1.46 and 1.5, respectively. For comparison, the evanescent field of bare SMF was also shown in Section II. Obviously, the evanescent field of tapered fiber as shown in Section I was much higher than bare SMF. The mirror image in Section I was caused by the reflection of slide glass.
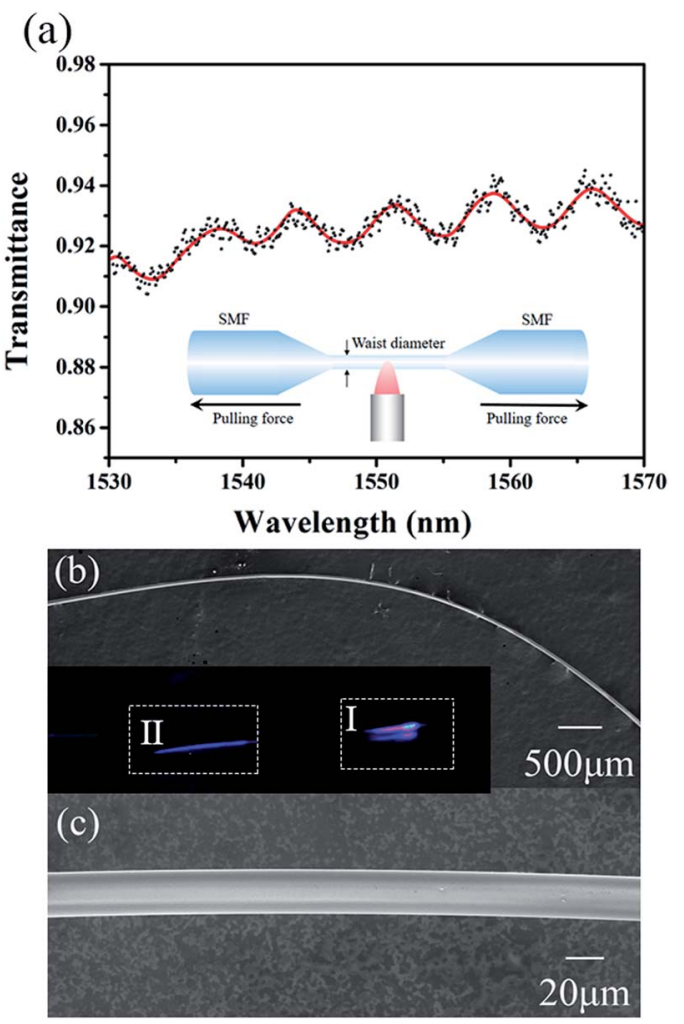

Fig. 1 (a) The transmission spectra of $25 \mu \mathrm{m}$-waist-diameter microfiber. Inset is the fabrication and structure of microfiber. SEM image of microfibers with waist diameter of $25 \mu \mathrm{m}$ in (b) $500 \mu \mathrm{m}$ scale bar and (c) $20 \mu \mathrm{m}$ scale bar. The inset is evanescent field of microfiber observed by a visible $455 \mathrm{~nm}$ light.

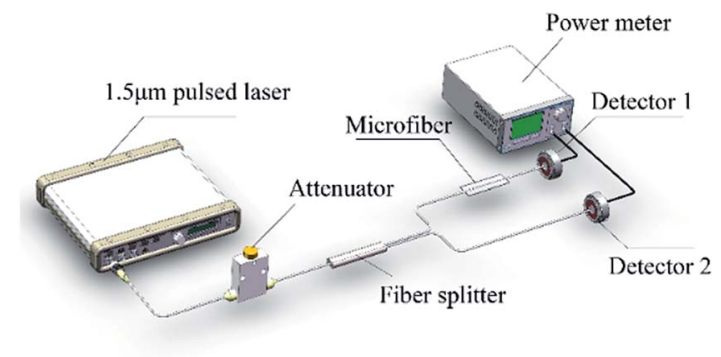

Fig. 2 Experimental setup for nonlinear absorption measurement of microfibers.

Open-aperture Z-scan and I-scan are the common techniques for the test of nonlinear optical absorption of materials. Several advantages exit in performing I-scan over the Z-scan technique, such as fully fiber-integrated setup or thin-enough sample. ${ }^{19}$ We measured the nonlinear optical absorption of microfiber by I-scan technique firstly, as described in Fig. 2. The probe laser is a self-constructed mode-locked fiber laser centered at $1557 \mathrm{~nm}$, with the pulse width and repetition rate of 750 fs and $15.6 \mathrm{MHz}$, respectively. The laser with maximum output power of $50 \mathrm{~mW}$ can be adjust by an attenuator. After that, the pulse was split equally with a fiber coupler, in which one branch performed as a reference beam and the other was connected with the prepared microfiber. By comparing the pulse intensities of the two branches, the transmissions versus the pulse intensity were obtained. The results are as described in Fig. 3. The experimental data were fitted according to the equation: ${ }^{20}$

$$
T=A \exp \left(\frac{-\Delta R}{1+I / I_{\mathrm{sat}}}\right)
$$

where $A$ is a normalization constant, $T$ is the transmission of microfiber, $I$ is the incident intensity, $\Delta R$ and $I_{\text {sat }}$ are the modulation depth and saturation intensity. The related

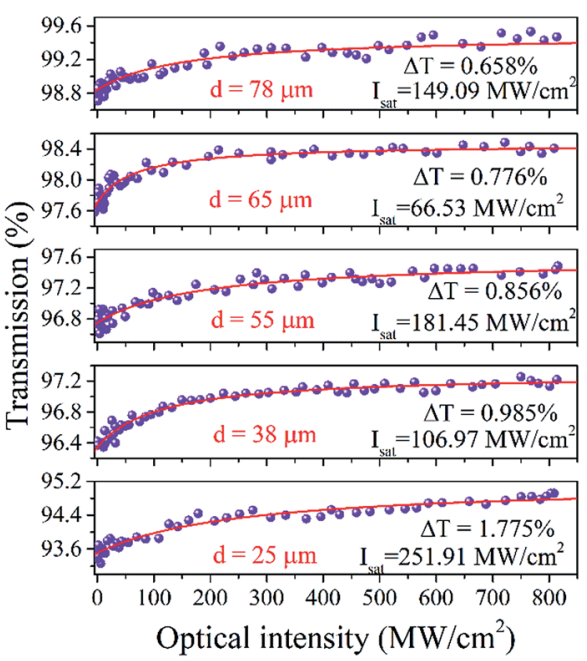

Fig. 3 Transmittance of microfibers as a function of pulse intensity with different waist diameters. 
Table 1 Detail results of nonlinear properties of microfibers with different waist diameter

\begin{tabular}{llll}
\hline $\begin{array}{l}\text { Waist diameter } \\
(\mu \mathrm{m})\end{array}$ & $\begin{array}{l}\text { Non-saturable } \\
\text { losses }\end{array}$ & $\begin{array}{l}\text { Modulation } \\
\text { depth }\end{array}$ & $\begin{array}{l}\text { Saturation intensity } \\
\left(\mathrm{MW} \mathrm{cm}^{-2}\right)\end{array}$ \\
\hline 78 & $0.59 \%$ & $0.66 \%$ & 149.09 \\
65 & $1.55 \%$ & $0.78 \%$ & 66.53 \\
55 & $2.56 \%$ & $0.86 \%$ & 181.45 \\
38 & $3.78 \%$ & $0.98 \%$ & 106.79 \\
25 & $5.11 \%$ & $1.78 \%$ & 251.91
\end{tabular}

goodness-of-fit $\left(R^{2}\right)$ is $90.53 \%, 91.52 \%, 91.29 \%, 96.67 \%, 94.71 \%$, respectively. Details were shown in Table 1 . The greatest contribution to the transmission loss of microfiber comes from the surface imperfections, cracks, and impurities connected with the microfiber surface. Obviously, the non-saturable loss increases with the decreasing of the waist diameter under the same manufacturing technique. The trend is in good agreement with precious reports on the propagation loss of microfibers. ${ }^{21}$ To our amusement, the modulation depth is inversely proportional to waist diameter of microfiber, which is an interesting conclusion on the properties of microfibers. However, the saturation intensity did not significantly laws with the change of the waist diameter of microfibers. We surmise the reason may be caused by the measurement error and fitting error.

Considering the enough large waist diameter $(25 \mu \mathrm{m})$ of microfiber compared to the light wavelength $(1.55 \mu \mathrm{m})$, the three layered tapered fiber model was adopted, as shown in Fig. 4(a). Combined Maxwell equations with boundary condition, the eigenvalue equations of $\mathrm{HE}_{\mathrm{mn}}$ modes could be described as following formula: $:^{22,23}$

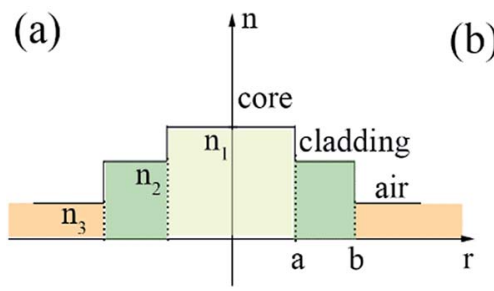

(b)

Fig. 4 (a) Index profile of the tapered fiber. (b) The radial field distribution of $\mathrm{HE}_{11}$ modes of microfiber with the diameter of $25 \mu \mathrm{m}$.

$$
X_{1}=n_{1} u_{1}{ }^{2} w_{2}{ }^{3} k^{2} a^{4} / m^{2} \beta^{2} V_{1}{ }^{2}, \quad X_{2}=n_{2} w_{2}{ }^{2} w_{3}{ }^{3} k^{2} b^{4} / m^{2} \beta^{2} V_{2}{ }^{2}
$$

$J_{\mathrm{m}}$ is the Bessel function of the first kind, $I_{\mathrm{m}}$ and $K_{\mathrm{m}}$ are the modified Bessel function of the first kind and second kind, respectively. $k_{0}=2 \pi / \lambda, a, b, n_{1}$ and $n_{2}$ are radius and refractive indices of core and the cladding of microfiber, $n_{3}$ is the refractive index in air, $\beta$ and $\lambda$ are propagation constant and the wavelength of light, respectively. According to the optical waveguide theory, the radial field can be described as follows:

$$
R(r)=\left\{\begin{array}{c}
A_{0} J_{\mathrm{m}}\left(U_{1} \frac{r}{a}\right) \quad 0 \leq r \leq a \\
A_{1} I_{\mathrm{m}}\left(W_{1} \frac{r}{b}\right)+A_{2} K_{\mathrm{m}}\left(W_{1} \frac{r}{b}\right) \quad a<r \leq b \\
A_{3} K_{\mathrm{m}}\left(W_{2} \frac{r}{b}\right) \quad r>b
\end{array}\right.
$$

By solving eqn (3), Fig. 4(b) shows the power distribution of $\mathrm{HE}_{11}$ mode of the tapered fiber with the diameters of $25 \mu \mathrm{m}$. It is clear that a part of energy guided outside of the cladding of microfibers as evanescent waves. So we guess the reason for

$$
\begin{aligned}
W_{0}^{2} & -\frac{2 X_{1} X_{2} n_{2}^{2}}{\left(w_{2}^{2} a b\right)^{2} n_{1} n_{3}}+X_{1}^{2} X_{2}^{2}\left[Y_{\mathrm{m}}\left(U_{\mathrm{m}} W_{0}+\frac{W_{1}}{w_{2} b}\right)+\frac{U_{\mathrm{m}} W_{2}+W_{3} / w_{2} b}{w_{2} a}\right]\left[Y_{\mathrm{m}}\left(U_{\mathrm{m}} W_{0}+\frac{n_{2}{ }^{2} W_{1}}{n_{3}{ }^{2} w_{2} b}\right)+\frac{n_{2}{ }^{2}}{n_{1}{ }^{2} w_{2} a}\left(U_{\mathrm{m}} W_{2}+\frac{n_{2}{ }^{2} W_{3}}{n_{3}{ }^{2} w_{2} b}\right)\right] \\
& -X_{1}^{2}\left(Y_{\mathrm{m}} W_{0}+\frac{W_{2}}{w_{2} a}\right)\left(Y_{\mathrm{m}} W_{0}+\frac{n_{2}{ }^{2} W_{2}}{n_{1}{ }^{2} w_{2} a}\right)-X_{2}^{2}\left(U_{\mathrm{m}} W_{0}+\frac{W_{1}}{w_{2} b}\right)\left(U_{\mathrm{m}} W_{0}+\frac{n_{2}^{2} W_{1}}{n_{3}{ }^{2} w_{2} b}\right)=0
\end{aligned}
$$

where,

$W_{0}=I_{\mathrm{m}}\left(w_{2} b\right) K_{\mathrm{m}}\left(a w_{2}\right)-I_{\mathrm{m}}\left(a w_{2}\right) K_{\mathrm{m}}\left(w_{2} b\right), \quad u_{1}=\left(n_{1}{ }^{2} k^{2}-\beta^{2}\right)^{1 / 2}$,

$W_{1}=I_{\mathrm{m}}\left(a w_{2}\right) K_{\mathrm{rh}}^{\prime}\left(w_{2} b\right)-I_{\mathrm{m}}^{\prime}\left(w_{2} b\right) K_{\mathrm{m}}\left(a w_{2}\right), \quad w_{2}=\left(\beta^{2}-n_{2}^{2} k^{2}\right)^{1 / 2}$

$W_{2}=I_{\mathrm{m}}\left(w_{2} b\right) K_{\mathrm{m}}^{\prime}\left(a w_{2}\right)-I_{\mathrm{m}}^{\prime}\left(a w_{2}\right) K_{\mathrm{m}}\left(w_{2} b\right), \quad w_{3}=\left(\beta^{2}-n_{3}^{2} k^{2}\right)^{1 / 2}$

$W_{3}=I_{\mathrm{m}}^{\prime}\left(w_{2} b\right) K_{\mathrm{m}}^{\prime}\left(a w_{2}\right)-I_{\mathrm{m}}^{\prime}\left(a w_{2}\right) K_{\mathrm{m}}^{\prime}\left(w_{2} b\right), \quad V_{1}=k a\left(n_{1}{ }^{2}-n_{2}{ }^{2}\right)^{1 / 2}$

$Y_{\mathrm{m}}=\frac{J_{\mathrm{m}}^{\prime}\left(a u_{1}\right)}{a u_{1} J_{\mathrm{m}}\left(a u_{1}\right)}, \quad U_{\mathrm{m}}=\frac{K_{\mathrm{m}}^{\prime}\left(w_{3} b\right)}{w_{3} b K_{\mathrm{m}}\left(w_{3} b\right)}, \quad V_{2}=k b\left(n_{2}{ }^{2}-n_{3}{ }^{2}\right)^{1 / 2}$ microfibers being a SA may be the evanescent field effect. Further investigation will be necessary.

\section{Experimental setup}

To test the saturable absorption ability of microfibers, we proposed a compact fully fiberized cavity, as schematically shown in Fig. 5. A $976 \mathrm{~nm}$ laser diode (LD) with maximum power of $500 \mathrm{~mW}$ was coupled into the gain medium via a 976/ 1550 wavelength division multiplexer (WDM). 0.9 m-highlydoped erbium fiber laser (EDF, LEKKI Er 110-4/125) was used with dispersion parameter of $-12 \mathrm{ps} \mathrm{nm}^{-1} \mathrm{~km}^{-1}$. A polarization-independent isolator (PI-ISO) was required to ensure unidirectional operation of the fiber laser. The polarization states of propagation light were rotated through a polarization controller (PC). The laser was coupled out through a $10 \%$ optical coupler (OC). The rest fiber of ring cavity, including the pigtails of microfiber and various components, 
were all SMF with dispersion parameter of $18 \mathrm{ps} \mathrm{nm}^{-1} \mathrm{~km}^{-1}$. The net dispersion was calculated to be about $-0.25 \mathrm{ps}^{2}$ with the total cavity length of $12.4 \mathrm{~m}$. The laser performance is monitored by an optical power meter (THORLABS, S148C), a 1 GHz digital oscilloscope (Tektronix DPO 7104) coupled with a 1 $\mathrm{GHz}$ photodetector, an optical spectrum analyzer (Yokogawa AQ6370C) and a $3 \mathrm{GHz}$ RF spectrum analyzer (Agilent N900A).

\section{Results and discussions}

First, to distinguish the proposed mode-locked fiber laser from the nonlinear polarization rotation scheme, ${ }^{24}$ the polarization property of microfiber using a polarized light with the wavelength of $1550 \mathrm{~nm}$ was test. By monitoring the change of output power through $25 \mu \mathrm{m}$-waist-diameter microfiber, obviously, the output power was almost no change. The result indicates that microfiber SA is polarization-independent. Then the ring cavity without inserting microfiber was executed. Only continuous wave (CW) could be observed when adjusted PC or increased pump power. The pump threshold of CW operation was $17 \mathrm{~mW}$ with slope efficiency of $8.19 \%$, as displayed in Fig. 6(a). After microfiber with waist diameter and taper length of $25 \mu \mathrm{m}$ and $15 \mathrm{~mm}$ incorporating, the threshold for CW laser increased up to $41 \mathrm{~mW}$ with the slope efficiency of $7.51 \%$. By slight adjusting the PC, the mode-locked fiber laser emerged when pump power further increased to $120 \mathrm{~mW}$. For better performance of modelocked fiber laser, the results were recorded when the pump power increased to $210 \mathrm{~mW}$. A typical pulse train is depicted in Fig. 6(b). Fundamental repetition rate was $16.59 \mathrm{MHz}$, which was determined by the cavity length of $12.4 \mathrm{~m}$ and verified the mode-locking state. The corresponding radio frequency (RF) spectrum was shown in Fig. 6(c), with resolution bandwidth $(\mathrm{RBW})$ of $20 \mathrm{kHz}$. The signal-to-noise $(\mathrm{S} / \mathrm{N})$ ratio was larger than $60 \mathrm{~dB}$, indicating good stability of mode-locked fiber laser based on microfiber. The results proved microfibers can be a good candidate as SA.

The emission spectrum is shown in Fig. 6(d). It can be clearly observed dual-wavelength mode-locked fiber laser with the peaks of 1593.9 and $1595.3 \mathrm{~nm}$ was generated. The two peaks were both Gaussian-like profile with the FWHM of 0.64 and $0.72 \mathrm{~nm}$, respectively. The spectrum showed clearly there was no Kelly sidebands, which may be caused by the spectral filtering effect. In this experiment, we didn't measure the real

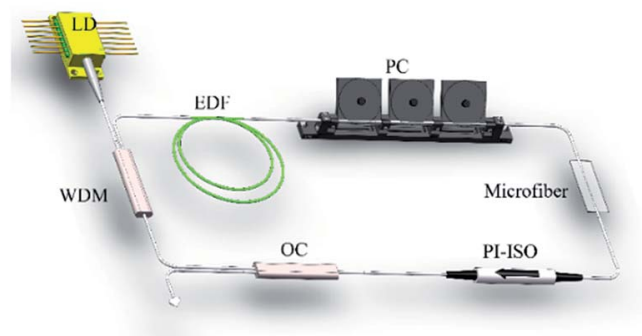

Fig. 5 Experimental setup of the mode-locked fiber laser based on microfibers.

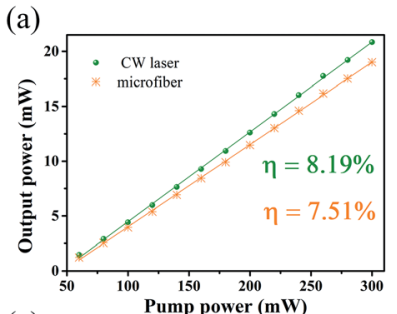

(c)
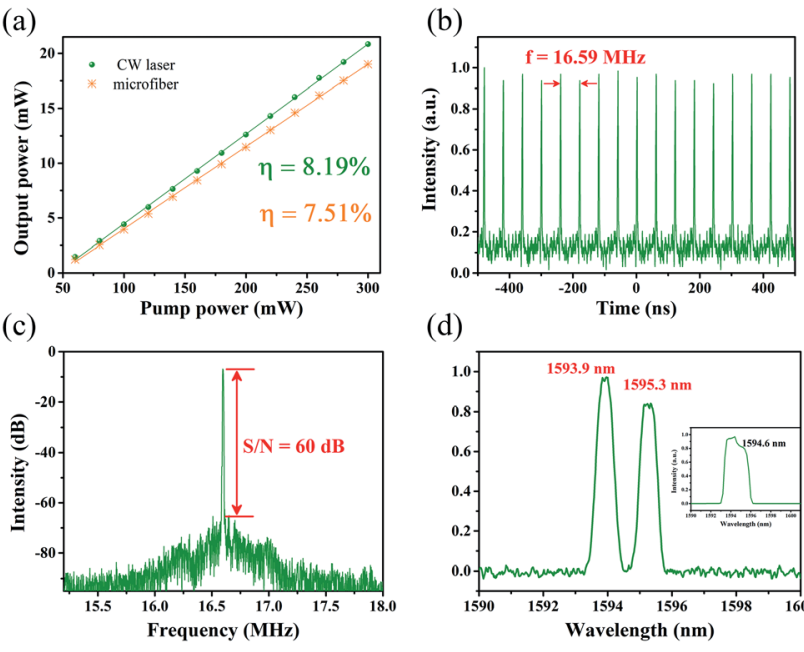

(d)

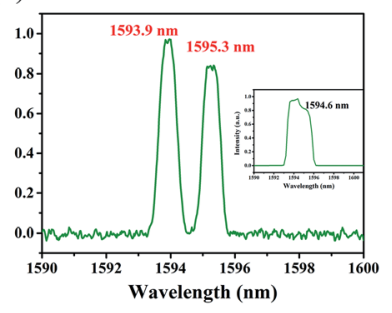

Fig. 6 Testing results of the microfi ber mode-locked fiber laser. (a) Output power as a function of pump power with (yellow) and without (green) microfiber. (b) Typical pulse train. (c) Radio frequency spectrum. (d) Spectrum, inset: laser spectrum without microfiber.

pulse width for the lack of suitable autocorrelator. However, according the soliton theory, the theoretical limit pulse duration could be estimated. The two peaks of spectrum with different FWHMs implied two separate pulse trains. The pulse duration of the two pulses was calculated to be 4.18 ps and 3.72 ps, respectively. For comparison, the laser spectrum of $\mathrm{CW}$ operation was also recorded, as shown in the inset of Fig. 6(d). No obvious dual-spectrum was observed. So we can reasonably infer that the microfiber contributed to generation of dualwavelength laser, which can be explained as follows: microfiber in the cavity not only worked as a SA, but a Mach-Zehnder interferometer (MZI). The MZI as fiber comb filter is an excellent method for generate multi-wavelength laser. ${ }^{25}$

Continue to raise pump power to $220 \mathrm{~mW}$, a new type of stable pulse train emerged, as shown in Fig. 7(a). The repetition rate was $49.79 \mathrm{MHz}$, correspondent to third harmonic of fundamental repetition rate. The change of repetition rate only depends on the pump power. There is no need to rotate the PC. The RF spectrum was depicted in Fig. 7(b), with the RBW of 4.7 $\mathrm{kHz}$. The $\mathrm{S} / \mathrm{N}$ ratio was higher than $55 \mathrm{~dB}$, indicating the harmonic mode-locked fiber laser was also in quite stable state. Adding the pump power to $250 \mathrm{~mW}$, the stable mode-locking fiber laser was collapsed suddenly. However, reducing the pump power again, the harmonic and fundamental modelocking pulses can be both reconstructed. As a result, the microfiber was confirmed owning a fairly high damage threshold.

Besides, the repeat scans of spectra measured within a span of a week were also recorded, as illustrated in Fig. 8. It can be verified that the mode-locked fiber laser based on microfiber with suitable diameter has a fairly long-term stability. The results proved the unique superiority of microfiber on the application to ultrafast fiber laser. We also demonstrated the same cavity with other prepared microfibers with different waist diameter. Except the $25 \mu \mathrm{m}$-diameter-microfiber, there is no 
(a)

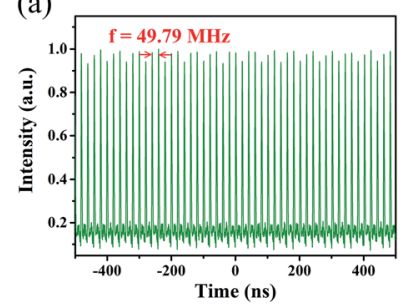

(b)

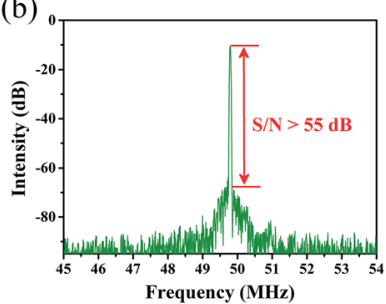

Fig. 7 Testing results of harmonic mode-locked fiber laser based on microfiber. (a) Typical pulse train. (b) Radio frequency spectrum.

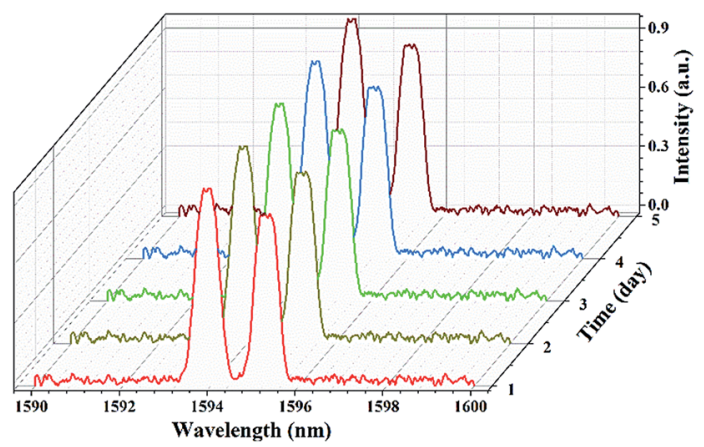

Fig. 8 The measured spectra of fundamental mode-locked fiber laser at 1 day interval within a span of a week.

stable mode-locking fiber laser, regardless of how to rotate PC or change pump power. The result displayed the waist diameter of microfiber is a main factor for the application as a SA. However, microfibers with smaller-diameters have not been fabricated due to the restriction of preparation. It is still hard to make deterministic conclusion on the selection of microfiber diameter as a SA. Specific origins of saturable absorption of microfibers are not so clear. Further detailed investigation will be essential.

\section{Conclusions}

In conclusion, a series of high-quality microfibers with different waist diameter was prepared by using flame-brushing technique. The saturable absorption property of microfibers were studied with I-scan measurement. The non-saturable loss and modulation depth both decreased with the increase of diameter of microfibers. Based on the microfiber with suitable diameter, an all-fiber-structure dual-wavelength mode-locked fiber laser was achieved. To our best of knowledge, it is the first time to investigate the optical modulation of microfiber and application to the ultrafast fiber laser. The long-term stability and repetition rate third harmonic mode-locked laser have fully testified microfiber could be a kind of excellent SAs for generating pulsed lasers.

\section{Conflicts of interest}

There are no conflicts to declare.

\section{Acknowledgements}

This work is supported by National Key Research and Development Program of China (2017YFB0405204).

\section{References}

$1 \mathrm{X} . \mathrm{Wu}$ and L. Tong, Nanophotonics, 2013, 2, 407-428.

2 G. Brambilla, J. Opt., 2010, 12, 043001.

3 L. Tong, F. Zi, X. Guo and J. Lou, Opt. Commun., 2012, 285, 4641-4647.

4 K. Lim, H. Ahmad, H. Arof and S. Harun, Fabrication and applications of microfiber, INTECH Open Access Publisher, 2012.

5 C. Caspar and E.-J. Bachus, Electron. Lett., 1989, 25, 15061508.

6 X. Li and H. Ding, Opt. Lett., 2012, 37, 5187-5189.

7 M. Sumetsky, Opt. Express, 2004, 12, 2303-2316.

8 L. Tong, J. Lou, R. R. Gattass, S. He, X. Chen, L. Liu and E. Mazur, Nano Lett., 2005, 5, 259-262.

9 J. Wo, G. Wang, Y. Cui, Q. Sun, R. Liang, P. P. Shum and D. Liu, Opt. Lett., 2012, 37, 67-69.

10 X. Jiang, Q. Yang, G. Vienne, Y. Li, L. Tong, J. Zhang and L. Hu, Appl. Phys. Lett., 2006, 89, 143513.

11 X. Jiang, Q. Song, L. Xu, J. Fu and L. Tong, Appl. Phys. Lett., 2007, 90, 233501.

12 S. G. Leon-Saval, T. A. Birks, W. J. Wadsworth, P. S. J. Russell and M. W. Mason, Opt. Express, 2004, 12, 2864-2869.

13 Y. Xu, X. Chen and Y. Zhu, Opt. Express, 2008, 16, 9205-9212. 14 Z. Sun, A. Martinez and F. Wang, Nat. Photonics, 2016, 10, 227-238.

15 K. Kashiwagi and S. Yamashita, Opt. Express, 2009, 17, 18364-18370.

16 W. Li, B. Chen, C. Meng, W. Fang, Y. Xiao, X. Li, Z. Hu, Y. Xu, L. Tong, H. Wang, W. Liu, J. Bao and Y. R. Shen, Nano Lett., 2014, 14, 955-959.

17 J. Zheng, X. Tang, Z. Yang, Z. Liang, Y. Chen, K. Wang, Y. Song, Y. Zhang, J. Ji, Y. Liu, D. Fan and H. Zhang, Adv. Opt. Mater., 2017, 5, 1700026.

18 G. Brambilla, F. Xu, P. Horak, Y. Jung, F. Koizumi, N. P. Sessions, E. Koukharenko, X. Feng, G. S. Murugan, J. S. Wilkinson and D. J. Richardson, Adv. Opt. Photonics, 2009, 1, 107-161.

19 R. Woodward and E. Kelleher, Appl. Sci., 2015, 5, 1440-1456.

20 S. Wang, H. Yu, H. Zhang, A. Wang, M. Zhao, Y. Chen, L. Mei and J. Wang, Adv. Mater., 2014, 26, 3538-3544.

21 A. V. Kovalenko, V. N. Kurashov and A. V. Kisil, Opt. Express, 2008, 16, 5797-5806.

22 H.-G. Unger, Planar Optical Waveguides and Fibers, Clarendon Press, 1997, pp. 310-320.

23 Z. Zhang and W. Shi, J. Opt. Soc. Am. A, 2005, 22, 2516-2526.

24 Z. Zhang, J. Gan, T. Yang, Y. Wu, Q. Li, S. Xu and Z. Yang, Opt. Lett., 2015, 40, 784-787.

25 M. I. Md Ali, S. A. Ibrahim, M. H. Abu Bakar, A. S. M. Noor, S. B. Ahmad Anas, A. K. Zamzuri and M. A. Mahdi, IEEE Photonics J., 2014, 6, 1-9. 